\title{
Tunable Collective Dynamics of Active Inclusions in Viscous Membranes
}

\author{
Harishankar Manikantan* \\ Department of Chemical Engineering, University of California, Davis, CA 95616, USA
}

\begin{abstract}
We study hydrodynamic interactions and clustering mechanisms of active membrane inclusions within lipid bilayers. Pairs of inclusions display unique oscillatory dynamics that disappear when the 3D fluid adjacent to the membrane is confined. We reduce the governing equations to a coupled dynamical system whose phase behavior reveals the striking role of bulk confinement in enhancing cluster formation within the membrane. Using numerical simulations, we then extend this finding to demonstrate the role of confinement in controlling large-scale aggregation of membrane inclusions.
\end{abstract}

Author's final version of article published in Phys. Rev. Lett. 125, 268101 (2020), doi:10.1103/PhysRevLett.125.268101

Biological membranes separate the cell cytoskeleton from the extracellular environment. The typical eukaryotic cell membrane is a crowded assembly of protein machines, molecular motors, ion channels, and other biomolecules embedded in a phospholipid bilayer matrix. This complex system forms a fluid 'mosaic' [1, 2]: membrane inclusions are effectively suspended in a quasi-2D fluid. Hydrodynamic models for the mobility of inclusions [3-8], together with the Einstein-Smoluchowski relation, have been widely successful at quantifying lateral diffusion of proteins [9], lipid domains [10, 11] and colloidal particles [12].

However, molecular machines are not passive inclusions: they operate as motors, manipulators and ion pumps, converting chemical energy to mechanical work. Active biomolecular machines achieve this via conformational changes [13, 14], polymerization [15], cyclical binding-unbinding transitions [16], lateral reorganization $[17,18]$ or rotation [19-21]. Importantly, inertia is negligible relative to viscous forces at these length and time scales: membrane inclusions are thus force- and torquefree. To leading order, active inclusions therefore induce hydrodynamic flows within the membrane (the 'interface') and in the surrounding 3D fluid (the 'subphase') via force or torque dipoles. Indeed, recent studies have confirmed the dipolar nature of the stresses exerted by active membrane proteins [14].

These hydrodynamic fields decay algebraically, setting up long-ranged disturbances that enhance diffusive transport $[16,22]$. Additionally, active membrane inclusions are rarely isolated and any disturbance induced by one perturbs its neighbor, leading to large-scale collective dynamics. For instance, rotor proteins such as ATP synthase were recently shown to self-assemble into a lattice state due to 'hydrosteric' interactions [21]. Recent evidence indicates that microtubule motors like kinesin and dynein coordinate their action to cluster on fluid membranes so that their collective dynamics drive faster or more efficient cellular transport [23-25]. However, a theoretical description of the membrane-mediated hydrodynamic interactions between inclusions that exert force dipoles to drive motion is still lacking.
In this work, we illustrate for the first time the nontrivial and coordinated motion of pairs of active force dipoles on viscous membranes. We first examine the phase behavior of a pair of hydrodynamically interacting membrane inclusions. Based on insights generated by the pair problem, we will illustrate strategies to experimentally manipulate long-range hydrodynamic interactions to enhance or suppress their aggregation. The key result of this work is illustrated in Fig. 1: confining the $3 \mathrm{D}$ subphase (e.g. via ultra-thin polymer supports [26]) modifies flow in the plane of the membrane in a manner that promotes aggregation of active dipoles. Traditional engineering of foreign inclusions in membranes [27, 28] has targeted interactions due to capillarity, curvature and electrostatics. In what follows, we propose an additional controllable parameter to tune collective motility and aggregation on lipid membranes.

The paradigmatic fluid mechanical model for a biological membrane [3-8] consists of a 2D manifold of surface viscosity $\eta_{s}$ atop a 3D fluid of viscosity $\eta$. Lipid molecules that comprise the bilayer are free to flow within
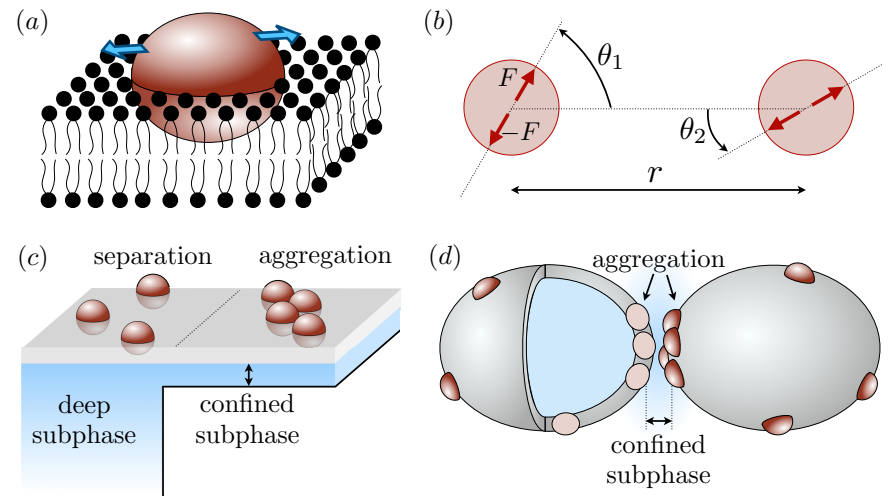

FIG. 1. (a,b) An active membrane inclusion exerts a force dipole, generating disturbance flows that perturb neighboring inclusions. Strategies to exploit hydrodynamics to aggregate membrance inclusions by confining the bulk phase via: (c) thin-film-supported bilayers; and (d) close confinement between adjacent cells or between a cell and a substrate or biopolymer. 
the 2D manifold, but exchange momentum with the adjacent subphase. Membrane inclusions are embedded within and constrained to move along the $2 \mathrm{D}$ quasi-fluid. We will assume that the bilayer is flat and that membrane curvature is negligible on length scales comparable to inclusions. Additionally, we will only account for subphase on one side of the interface for simplicity: presence of a 3D fluid on both sides will introduce a multiplicative factor in the Green's functions below, and do not qualitatively change the results.

Stress balance on a viscous 2D manifold is prescribed by the Boussinesq-Scriven equations [29, 30]. Let $\mathbf{u}(\mathbf{r})$ be the in-plane fluid velocity at a point $\mathbf{r}=(x, y)$ on a planar membrane represented by $z=0$. Phospholipid bilayers (indeed, all insoluble surfactant-laden interfaces [30]) are 2D incompressible so that $\boldsymbol{\nabla}_{s} \cdot \mathbf{u}=0$, where $\boldsymbol{\nabla}_{s}=(\mathbf{I}-\hat{\mathbf{z}} \hat{\mathbf{z}}) \cdot \boldsymbol{\nabla}=(\partial / \partial x, \partial / \partial y)$ is the surface gradient operator. When perturbed by a point force $\mathbf{F}$ at the origin, the surface stress balance is

$$
\boldsymbol{\nabla}_{s} \Pi=\eta_{s} \nabla_{s}^{2} \mathbf{u}-\left.\eta \frac{\partial \mathbf{v}}{\partial z}\right|_{z=0}+\mathbf{F} \delta(\mathbf{r})
$$

where $\Pi(\mathbf{r})$ is the surface pressure. Equation (1) couples to the $3 \mathrm{D}$ fluid flow via the viscous traction $\eta \partial \mathbf{v} / \partial z$ exerted at the interface. The bulk fluid velocity $\mathbf{v}(x, y, z)$ satisfies the Stokes equations with the additional no-slip condition $\mathbf{v}=\mathbf{u}$ at $z=0$. The surface disturbance velocity following Eq. (1) can be determined by solving this coupled system to obtain

$$
\mathbf{u}=\mathbf{G}(\mathbf{r}) \cdot \mathbf{F}=\frac{1}{4 \pi \eta_{s}}\left[A(r) \mathbf{I}+B(r) \frac{\mathbf{r r}}{r^{2}}\right] \cdot \mathbf{F},
$$

where $A(r)$ and $B(r)$ with $r=|\mathbf{r}|$ depend on the position, the geometry, and the Saffman-Delbrück length $\ell=\eta_{s} / \eta$ $[8,30-32]$. If the subphase $3 \mathrm{D}$ fluid is unbounded (the 'deep-subphase' limit), $A(r)=\pi\left[H_{0}(d)-H_{1}(d) / d+\right.$ $\left.2 / \pi d^{2}-\left(Y_{0}(d)-Y_{2}(d)\right) / 2\right]$ and $B(r)=\pi\left[-H_{0}(d)+\right.$ $\left.2 H_{1}(d) / d-4 / \pi d^{2}-Y_{2}(d)\right]$, with $d=r / \ell$. Here, $H_{\nu}$ and $Y_{\nu}$ are the Struve and Bessel functions of the second kind of order $\nu$, respectively. Flow is dominated by surface viscous stresses and momentum transfer is restricted to the plane of the membrane when $r \ll \ell$. Conversely, $3 \mathrm{D}$ momentum transfer and bulk viscous traction dominate flow at distances $r \gg \ell$.

However, motor proteins (or surface-attached swimmers and catalysts) are force-free inclusions. The disturbance flow created by such inclusions can be obtained by perturbing the Boussinesq-Scriven equation with the appropriate stresslet [33] or by integrating over appropriate force densities [34,35]. An easier and more intuitive approach for small inclusions identifies the leading-order disturbance flow as that arising from a dipole (Fig. 1), constructed by two equal and opposite point forces separated by a distance $L$. This distance is characteristic of typical inclusion dimensions, so $L=O(10 \mathrm{~nm})$.


FIG. 2. Fluid streamlines in the plane of the membrane around a force dipole on an unconfined subphase when (a) $r>\ell$ and (b) $r<\ell$, where $\ell$ is the Saffman-Delbrück length. (c) Confinement introduces azimuthal streamlines over distances smaller than $\ell$ if $r>\ell_{c}$ with $\ell_{c}=\sqrt{\ell H}<\ell$.

Following this approach, the surface velocity at a location $\mathbf{r}$ on the membrane due to an inclusion oriented in the direction $\hat{\mathbf{p}}$ and located at the origin is $\mathbf{u}=$ $\mathbf{G}(\mathbf{r}-L \hat{\mathbf{p}} / 2) \cdot \mathbf{F}-\mathbf{G}(\mathbf{r}+L \hat{\mathbf{p}} / 2) \cdot \mathbf{F}$. Owing to small size of $O(\mathrm{~nm})$ inclusions relative to flow over $O(\mu \mathrm{m})$ length scales, we retain only the leading-order terms in the Taylor expansion of $\mathbf{G}$ to get

$$
u_{j}=-\sigma p_{i} \frac{\partial G_{j k}}{\partial x_{i}} p_{k}
$$

Here, $\sigma=F L$ is the magnitude of the stresslet corresponding to the dipolar inclusion. Much like with 3D swimmers [36], $\sigma>0$ denotes an in-plane 'pusher' which pushes fluid in the directions $\pm \hat{\mathbf{p}}$, whereas a 'puller' with $\sigma<0$ creates inward flows along $\pm \hat{\mathbf{p}}$.

We wish to highlight the role of the subphase fluid in determining inter-particle interactions on the interface. Phospholipid bilayers are typically highly surface viscous with $\eta_{s} \sim 10^{-9} \mathrm{Ns} / \mathrm{m}$ so that the Saffman-Delbrück length is $\ell \sim 10 \mu \mathrm{m}$. The system is subphase-dominated at length scales larger that $\ell$. The Green's function then simplifies with $A(r) \rightarrow 2 \ell^{2} / r^{2}$ and $B(r) \rightarrow 2 \ell / r-4 \ell^{2} / r^{2}$. Then, the disturbance flow due to a dipole, using Eq. (3), is

$$
\mathbf{u}_{r>\ell}^{\text {deep }}=-\frac{\sigma}{2 \pi \eta r^{2}}\left[\left(1-3 \cos ^{2} \theta\right) \hat{\mathbf{r}}+\cos \theta \hat{\mathbf{p}}\right],
$$

where $\hat{\mathbf{r}}$ is the radial unit vector relative to the membrane inclusion at the origin and $\theta=\hat{\mathbf{r}} \cdot \hat{\mathbf{p}}$. This flow field is illustrated in Fig. 2(a).

By contrast, $A(r) \rightarrow \log (\ell / r)$ and $B(r) \rightarrow 1$ to leading order when $r \ll \ell$. The Green's function is then indeed the $2 \mathrm{D}$ stokeslet and the flow diverges unless regularized by bulk viscous traction at length scales above $\ell[3]$. However, the dipolar flow field from Eq. (3) in this limit is well-defined:

$$
\mathbf{u}_{r<\ell}^{\text {deep }}=-\frac{\sigma}{4 \pi \eta_{s} r}\left(1-2 \cos ^{2} \theta\right) \hat{\mathbf{r}} .
$$

A significant difference emerges in surface-dominated systems relative to Eq. (4): the surface disturbance flow is entirely along the radial direction when $r \ll \ell$ (Fig. 2(b)). 



FIG. 3. (a) Distance between a pair of inclusions and (b) dipolar orientations in an unconfined membrane, obtained by integrating Eqs. (7)-(8). Shaded regions indicate times when inclusions approach each other. (c) Phase behavior and dynamical flow lines of $\left(\theta_{1}, \theta_{2}\right)$ at fixed $r$. The solid line is the closed orbit corresponding to the initial conditions in (a-b). Pairs aggregate when $\dot{r}<0$ (shaded) and separate otherwise. (d) Inter-inclusion separation and (e) orientations in a confined membrane, following Eqs. (9)-(10). (f) The corresponding phase portrait shows that oscillations are no longer possible. The solid line shows the pair in (d-e) aggregate upon being drawn into the region of attraction $(\dot{r}<0$, shaded).

The azimuthal component of disturbance flows causes membrane inclusions to aggregate more easily, as we show below. However, azimuthal streamlines emerge only when $r \gg \ell \sim 10 \mu \mathrm{m}$. It is therefore of practical interest to manipulate the cut-off length $\ell$. Tuning the viscosity of the suspending fluid is one option; however, this strategy can at most reduce $\ell$ by an $O(1)$ factor. The novel insight of this work is that an alternative (and experimentally accessible) strategy to enhance the viscous drag from the bulk fluid - and therefore introduce azimuthal flow in the membrane plane is to confine the depth $H$ of the $3 \mathrm{D}$ bulk phase adjacent to the membrane. Using the methods of lubrication theory for a thin subphase layer $[6,31,32]$, it can be shown that the coefficients in the Green's function satisfying Eq. (1) are $A(r)=-2 / d_{c}^{2}+2 K_{0}\left(d_{c}\right)+2 K_{1}\left(d_{c}\right) / d_{c}$ and $B(r)=4 / d_{c}^{2}-2 K_{0}\left(d_{c}\right)-4 K_{1}\left(d_{c}\right) / d_{c}$, where $K_{\nu}$ is the modified Bessel function of order $\nu$. In this case, $d_{c}=r / \ell_{c}$ where $\ell_{c}=\sqrt{\ell H}$ is a modified cut-off length beyond which bulk viscous effects become dominant.

Thus, reducing subphase depth $H$ has the effect of screening the Saffman-Delbrück length $\ell$ : confinement constrains surface viscous stresses to be sub-dominant beyond a length scale $\ell_{c}<\ell$ if $H \lesssim 10 \mu \mathrm{m}$. In close confinement between adjacent vesicles or atop a thin film (as illustrated in Fig. 1) or polymer cushions such that $H \sim 10-100 \mathrm{~nm}[26]$, the modified cut-off distance can be $\ell_{c}<1 \mu \mathrm{m}$. At distances greater than this modified lower cutoff, $A(r) \rightarrow-2 \ell H / r^{2}$ and $B(r) \rightarrow 4 \ell H / r^{2}$. Unsurprisingly, the Green's function then represents a potential dipole, which is the singularity corresponding to a point force in a confined Hele-Shaw system. The flow flow corresponding to a force-free membrane inclusion, following Eq. (3), then becomes

$$
\mathbf{u}_{r>\ell_{c}}^{\text {confined }}=-\frac{\sigma H}{\pi \eta r^{3}}\left[\left(1-4 \cos ^{2} \theta\right) \hat{\mathbf{r}}+2 \cos \theta \hat{\mathbf{p}}\right],
$$

which is a potential quadrupole (Fig. 2(c)). Equation (6) re-introduces azimuthal flow lines on a viscous membrane at lengthscales much smaller than $\ell$ by amplifying bulk viscous stresses in the thin film underlying the interface. A membrane machine of size $O(10 \mathrm{~nm})$ generating local forces of $O(10 \mathrm{pN})$ has stresslet strength $\sigma \sim 10-100 k_{B} T$. When confined with $H \sim 100 \mathrm{~nm}$, Eq. (6) predicts $O(\mu \mathrm{m} / \mathrm{s})$ flows due to such an inclusion that can drive co-ordinated motion across cellular $O(\mu \mathrm{m})$ length scales.

We approach such large scale collective dynamics by first examining the hydrodynamic interactions between a pair of inclusions. Each inclusion translates with the disturbance velocity induced by the other. The interinclusion separation evolves as $\dot{r}=\left(\mathbf{u}_{21}-\mathbf{u}_{12}\right) \cdot \hat{\mathbf{r}}$, where $\mathbf{u}_{21}$ and $\mathbf{u}_{12}$ are translational velocities of inclusion 2 relative to 1 and vice versa. Assuming equal stresslet strengths $\sigma=\sigma_{1}=\sigma_{2}$, the distance between inclusions on an unconfined viscous membrane evolves as

$$
\dot{r}=\frac{\sigma}{2 \pi \eta_{s} r}\left(\cos ^{2} \theta_{1}+\cos ^{2} \theta_{2}-1\right) .
$$

Following fluid kinematics, each inclusion also rotates at a rate equal to half the vorticity of the disturbance field of the other: $\boldsymbol{\Omega}_{2}=\frac{1}{2} \boldsymbol{\nabla} \times \mathbf{u}_{21}$ and $\boldsymbol{\Omega}_{1}=\frac{1}{2} \boldsymbol{\nabla} \times \mathbf{u}_{12}$. The dipole orientations $\theta_{1}(t)$ and $\theta_{2}(t)$ relative to the line through the particle centers (Fig. 1) thus evolve as

$$
\dot{\theta}_{1}=-\frac{\sigma}{4 \pi \eta_{s} r^{2}} \sin 2 \theta_{2}, \quad \dot{\theta}_{2}=-\frac{\sigma}{4 \pi \eta_{s} r^{2}} \sin 2 \theta_{1} .
$$

This nonlinear coupling between $r, \theta_{1}$ and $\theta_{2}$ leads to the 
unique oscillatory behavior shown in Figure 3(a-b) and in the supplementary videos [37].

To examine these novel nonlinear dynamics, we map the phase portrait of the dynamical system governing $\left(\theta_{1}, \theta_{2}\right)$ at fixed $r$ in Fig. 3(c) . The phase 'flow' lines weave in and out of regions where $\dot{r}$ is negative (shaded) so that inclusions approach each other and where $\dot{r}$ is positive (unshaded) so that the inter-inclusion separation grows. Indeed, all but a limited class of initial conditions result in oscillations. The fixed points at the corners of the shaded region are nonlinear centers [38], so that a pair of inclusions starting at any of these centers maintain their relative orientation and separation. Any perturbation from these points would place them in the family of closed orbits around the centers, launching them into nonlinear oscillations like in Fig. 3(a-b). The fixed points at the corners of the phase portrait and at $(\pi / 2, \pi / 2)$ are hyperbolic saddle nodes: inclusions separate or approach each other when placed at these positions, but are again easily perturbed into nonlinear oscillations. Real inclusions have finite size, so oscillations are limited to cases when $r \gtrsim 2 L$ where $L$ is the particle size.

The frequency of these oscillations can be estimated by a linear stability analysis around the centers when the inclusions are initially at a separation $R_{0}$. Assuming that the angles $\theta_{1}$ and $\theta_{2}$ grow as $e^{\alpha t}$ when perturbed from one of the centers and solving for the eigenvalues of the corresponding Jacobian matrix gives $\alpha= \pm i \sigma / 2 \pi \eta_{s} R_{0}^{2}$. The growth rates are imaginary, consistent with oscillations that neither grow nor decay. The time period of these oscillations is then $T_{0}=2 \pi /|\alpha|=4 \pi^{2} \eta_{s} R_{0}^{2} / \sigma$. This approximation holds so long as the separation $r(t)$ remains close to $R_{0}$. The period of oscillations is therefore determined by the relative strengths of the characteristic force $\sim \sigma / R_{0}$ due to hydrodynamic interactions and the surface viscous resistance $\sim \eta_{s} R_{0}$.

Entirely different phase dynamics arise in a system of membrane inclusions confined by a thin subphase. Using Eq. (6), inter-inclusion separation now evolves as

$$
\dot{r}=\frac{2 \sigma H}{\pi \eta r^{3}}\left(\cos ^{2} \theta_{1}+\cos ^{2} \theta_{2}-1\right) .
$$

The local angular velocity as a result of the disturbance field is zero, which is not surprising as potential flow in a Hele-Shaw system is a vorticity-free. So the two particles translate without rotation in the lab frame. However, the disturbance velocity due to each particle has an azimuthal component $\mathbf{u}_{\perp}=\sin 2 \theta \hat{\boldsymbol{\theta}}$, which has the effect of rotating the line joining particle centers by an angle $\phi$. In the frame of reference moving with one of the particles and rotating with the line joining particle centers, each particle therefore experiences an angular rotation of magnitude $\dot{\phi}=\left|\mathbf{u}_{\perp}\right| / r$. The net rate of rotation in the moving frame is then

$$
\dot{\theta}_{1}=\dot{\theta}_{2}=\frac{\sigma H}{\pi \eta r^{4}}\left(\sin 2 \theta_{1}+\sin 2 \theta_{2}\right) .
$$

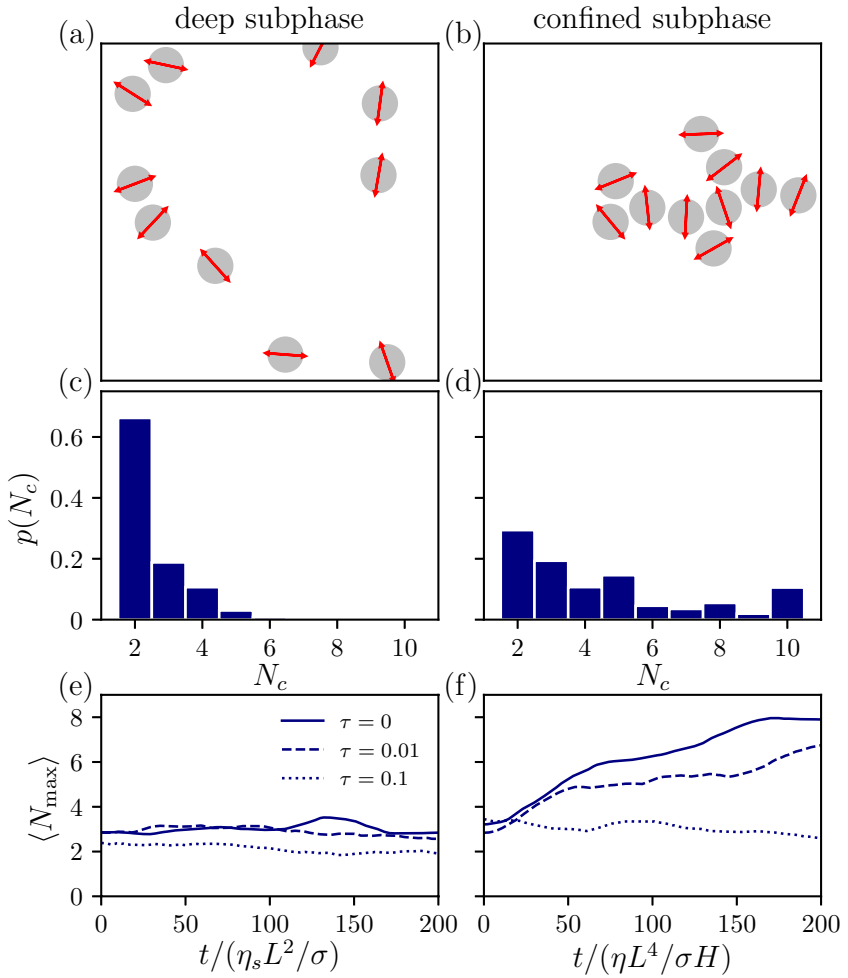

FIG. 4. (a-b) Snapshots from simulations of hydrodynamically interacting dipolar membrane inclusions in a $2 \mathrm{D}$ periodic domain. Arrows denote dipole orientations. Inclusions chaotically approach and separate when the bulk fluid is unconfined, but aggregate when confined. (c-d) Normalized distributions of number $N_{c}$ of inclusions in a cluster across 10 simulations up to 200 time steps each [37]. Small aggregates dynamically form and separate when unconfined, whereas larger aggregates are stable when confined. (e-f) Growth of the largest cluster of size $N_{\max }$ with time, ensemble averaged across simulations each. Hydrodynamic effects are indistinguishable from Brownian fluctuations for an unconfined subphase even when the effective temperature $\tau=k_{B} T / \sigma \rightarrow 0$. By contrast, clusters grows when the membrane is confined.

Figure $3(\mathrm{~d})$ shows the phase diagram of $\left(\theta_{1}, \theta_{2}\right)$ at fixed $r$ for a confined membrane, revealing that oscillatory dynamics are not possible when the subphase is confined. In fact, very few relative orientations are stable: any perturbation from the points where $\dot{\theta}_{1}=\dot{\theta}_{2}=0$ reorients inclusions in a manner that leads to a negative $\dot{r}$. In stark contrast to the unconfined geometry, pairs of inclusions are essentially trapped once within the region of attraction leading to cluster formation.

These differences are amplified in more crowded systems, as shown in Fig. 4 using simulations of 10 particles in a $10 L \times 10 L 2 \mathrm{D}$ periodic box; the qualitative results illustrated here were checked to be independent of box size [37]. In addition to hydrodynamic interactions, these simulations account for a short-range soft repulsion to prevent particle overlap. The repulsive force 
decays exponentially beyond a steric length $L$ : far-field co-ordinated motion is thus driven entirely by membrane hydrodynamic interactions via Eq. (5) or (6). The simulations also account for thermal fluctuations following the fluctuation-dissipation theorem [37]. The strength of stochastic Brownian forces relative to the disturbance flow experienced by an inclusion is quantified by an effective temperature $\tau=k_{B} T / \sigma$. Alternatively, $\tau^{-1}$ may be interpreted as a Péclet number $P e=\sigma / k_{B} t$ which compares convection on the surface due to dipolar flow relative to thermal diffusion.

As shown in Fig. 4, clusters do not form on membranes atop an unconfined or deep subphase. In this case, streamlines around each particle (Fig. 2(b)) are as likely to attract a pair of inclusions as they are to separate them. Thermal fluctuations and disturbances due to neighboring inclusions perturb a two-particle system away from fixed points and out of the region of attraction in the phase portrait in Fig. 3(c). In a multi-particle system, inclusions thus chaotically approach each other until a perturbation causes them to separate [37]. A quantitative index of the long-time aggregation behavior is the number $N_{\max }$ of particles in the largest cluster (Fig. 4(e)): even in the athermal limit $(\tau=0)$, aggregate sizes are statistically indistinguishable from a system dominated by Brownian fluctuations.

In stark contrast, collective hydrodynamics on a confined viscous interface always draw neighbors towards each other. Any relative configuration other than a headon orientation leads to trapped pairs. The head-on orientation is almost always perturbed by other inclusions (or thermal noise), and so aggregates are stable and the typical cluster size $N_{c}$ grows. Figure $4(\mathrm{~d})$ shows the distribution across simulations upto a dimensionless time of 200: smaller clusters at early times coalesce stably into large system-spanning aggregates [37]. The effective temperature is in the range of $\tau \sim 0.01$ for a membrane machine of size $O(10 \mathrm{~nm})$ generating local forces of $O(10-100 \mathrm{pN})$. The long-time effect of subphase confinement in this biologically relevant regime is clear from Fig. 4(f): the largest cluster continues growing due to confined hydrodynamics.

Note that hydrodynamic interactions become purely radial at small inter-inclusion separations even in the confined geometry (when $r \ll \ell_{c}$ ). However, the confinement depth $H$ can be controlled to ensure that the screened cut-off $\ell_{c}$ is small enough so that far-field hydrodynamics always promote aggregation, whereas shortrange finite-size effects and intermolecular interactions dominate at length scales smaller than $\ell_{c}$. The simulations are performed to highlight this limit, assuming that the bulk phase is sufficiently confined so that hydrodynamics are bulk-dominated at all inter-inclusion separations. In other words, $\ell_{c}$ is taken to be comparable to inclusion size $L$ so that the hydrodynamic disturbance felt by neighboring inclusions is always quadrupolar.
This dramatic shift due merely to subphase confinement can be exploited to accumulate active membrane inclusions by simply introducing a confining wall, or supporting the bilayer on a thin film or polymer cushion atop a substrate (Fig. 1(c)). Alternatively, bringing two vesicles in close proximity would thin the suspending fluid layer between them, mimicking the effect of subphase confinement on either membrane (Fig. 1(d)). Similarly, the subphase is locally confined when motors attach onto an adjacent macromolecule. Indeed, enhanced cargo transport has recently been reported due to clustering of membrane motors in confined regions near microtubular attachment $[24,25]$. Further, aggregates increase the effective surface viscosity of the membrane $[8,30]$, resulting in locally immobilized regions of the bilayer. Confinement thus provides a strategy to tune the local fluidity and, therefore, membrane diffusivity - of crowded phospholipid bilayers. Building on these insights, we anticipate that the present work will spur new directions of experimental inquiry into active and tunable membranes.

* hmanikantan@ucdavis.edu

[1] S. J. Singer and G. L. Nicolson, Science 175, 720 (1972).

[2] M. Bloom, E. Evans, and O. G. Mouritsen, Quarterly Reviews of Biophysics 24, 293 (1991).

[3] P. G. Saffman and M. Delbrück, Proceedings of the National Academy of Sciences 72, 3111 (1975).

[4] P. G. Saffman, Journal of Fluid Mechanics 73, 593 (1976).

[5] B. D. Hughes, B. A. Pailthorpe, and L. R. White, Journal of Fluid Mechanics 110, 349 (1981).

[6] E. Evans and E. Sackmann, Journal of Fluid Mechanics 194, 553 (1988).

[7] H. A. Stone and A. Ajdari, Journal of Fluid Mechanics 369, 151 (1998).

[8] N. Oppenheimer and H. Diamant, Biophysical Journal 96, 3041 (2009).

[9] A. Kusumi, C. Nakada, K. Ritchie, K. Murase, K. Suzuki, H. Murakoshi, R. S. Kasai, J. Kondo, and T. Fujiwara, Annual Review of Biophysics and Biomolecular Structure 34, 351 (2005).

[10] P. Cicuta, S. L. Keller, and S. L. Veatch, The Journal of Physical Chemistry B 111, 3328 (2007), 0611492.

[11] W. F. Zeno, M. O. Ogunyankin, and M. L. Longo, Biochimica et Biophysica Acta - Biomembranes 1860, 1994 (2018).

[12] V. Prasad, S. A. Koehler, and E. R. Weeks, Physical Review Letters 97, 176001 (2006), 0604262.

[13] J.-B. Manneville, P. Bassereau, D. Lévy, and J. Prost, Physical Review Letters 82, 4356 (1999).

[14] M.-J. Huang, R. Kapral, A. S. Mikhailov, and H.-Y. Chen, The Journal of Chemical Physics 138, 195101 (2013).

[15] A. Khondker, R. J. Alsop, and M. C. Rheinstädter, Membranes 7, 49 (2017).

[16] A. S. Mikhailov and R. Kapral, Proceedings of the National Academy of Sciences 112, E3639 (2015).

[17] S. Mayor and M. Rao, Traffic 5, 231 (2004). 
[18] K. Gowrishankar, S. Ghosh, S. Saha, R. C., S. Mayor, and M. Rao, Cell 149, 1353 (2012).

[19] H. C. Berg, Annual Review of Biochemistry 72, 19 (2003).

[20] W. Junge and N. Nelson, Annual Review of Biochemistry 84, 631 (2015).

[21] N. Oppenheimer, D. B. Stein, and M. J. Shelley, Physical Review Letters 123, 148101 (2019).

[22] Y. Hosaka, K. Yasuda, R. Okamoto, and S. Komura, Physical Review E 95, 052407 (2017).

[23] O. Campàs, C. Leduc, P. Bassereau, J. Casademunt, J.F. Joanny, and J. Prost, Biophysical Journal 94, 5009 (2008).

[24] R. Grover, J. Fischer, F. W. Schwarz, W. J. Walter, P. Schwille, and S. Diez, Proceedings of the National Academy of Sciences 113, E7185 (2016).

[25] A. Rai, D. Pathak, S. Thakur, S. Singh, A. K. Dubey, and R. Mallik, Cell 164, 722 (2016).

[26] M. Tanaka and E. Sackmann, Nature 437, 656 (2005).

[27] A. Šarić and A. Cacciuto, Soft Matter 9, 6677 (2013).

[28] S. Dasgupta, T. Auth, and G. Gompper, Journal of Physics: Condensed Matter 29, 373003 (2017).
[29] L. Scriven, Chemical Engineering Science 12, 98 (1960).

[30] H. Manikantan and T. M. Squires, Journal of Fluid Mechanics 892, P1 (2020).

[31] T. M. Fischer, Journal of Fluid Mechanics 498, 123 (2004).

[32] D. K. Lubensky and R. E. Goldstein, Physics of Fluids 8, 843 (1996), 9602002.

[33] G. Batchelor, Journal of fluid mechanics 41, 545 (1970).

[34] C. Alas, T. R. Powers, and T. Kuriabova, (2020), arXiv:2002.02106.

[35] N. G. Chisholm and K. J. Stebe, (2020), arXiv:2004.09564.

[36] E. Lauga and T. R. Powers, Reports on Progress in Physics 72, 096601 (2009).

[37] See Supplemental Material for details of the simulation method, and videos showing co-ordinated dynamics of pairs of inclusions, large scale aggregation, the effect of finite temperature, and the indifference to box size.

[38] S. H. Strogatz, Nonlinear Dynamics and Chaos: with Applications to Physics, Biology, Chemistry, and Rngineering (Westview press, 1994). 


\title{
Supplementary information: Tunable collective dynamics of active inclusions on viscous membranes
}

\author{
Harishankar Manikantan \\ Department of Chemical Engineering, University of California, Davis, CA 95616, USA
}

In the following, we outline the Brownian dynamics simulations used to verify large-scale clustering behavior (figure 4 and related discussion in the manuscript). Each inclusion $i$ translates and rotates due to the collective disturbance field of the other $N-1$ inclusions and due to Brownian fluctuations:

$$
\begin{aligned}
\frac{d \mathbf{x}_{i}}{d t} & =\sum_{j \neq i}^{N} \mathbf{u}_{j}\left(\mathbf{x}_{i}\right)+\mathbf{u}_{i}^{\mathrm{Br}}, \\
\frac{d \theta_{i}}{d t} & =\sum_{j \neq i}^{N} \dot{\theta}_{j}\left(\mathbf{x}_{i}\right)+\Omega_{i}^{\mathrm{Br}} .
\end{aligned}
$$

The velocities due to thermal fluctuations relate to the collective hydrodynamic mobilities [1] of the inclusion via the fluctuation-dissipation theorem. The concatenated $2 \mathrm{~N}$ vector $\mathcal{U}^{\mathrm{Br}}$ of the two velocity components of $\mathbf{u}^{\mathrm{Br}}$ of all $N$ inclusions satisfies

$$
\left\langle\mathcal{U}^{\mathrm{Br}}(t)\right\rangle=\mathbf{0}, \quad\left\langle\mathcal{U}^{\mathrm{Br}}(t) \mathcal{U}^{\mathrm{Br}}\left(t^{\prime}\right)\right\rangle=2 k_{B} T \mathcal{M} \delta\left(t-t^{\prime}\right) .
$$

Here, $\mathcal{M}$ is a $2 N \times 2 N$ grand mobility matrix with self-mobility of each inclusion along the diagonal, and the collective mobility due to hydrodynamic interactions populate the off-diagonal terms. Similar equations can be written for the rotational motion of each inclusion. The central $2 \times 2$ blocks along the diagonal are the dominant local mobilities of each inclusion and the off-diagonal terms are the sub-dominant interaction terms that become more relevant at higher concentration. Owing to the diluteness of our system and for simplicity, we only account for local mobilities that decouples the fluctuation-dissipation relation of each inclusion from the rest. The translational Brownian velocities then satisfy local relations

$$
\left\langle\mathbf{u}^{\mathrm{Br}}(t)\right\rangle=\mathbf{0}, \quad\left\langle\mathbf{u}^{\mathrm{Br}}(t) \mathbf{u}^{\mathrm{Br}}\left(t^{\prime}\right)\right\rangle=2 k_{B} T \mathbf{M} \delta\left(t-t^{\prime}\right),
$$

where $\mathbf{M}$ is a $2 \times 2$ tensor. Further, we will assume that each inclusion is a cylindrical disk of radius $L$ embedded within the monolayer, so that the mobility is isotropic in the plane of the membrane: $\mathbf{M}=M(\mathbf{I}-\hat{\mathbf{z}} \hat{\mathbf{z}})$. Similarly, rotational Brownian motion satisfies

$$
\left\langle\Omega^{\mathrm{Br}}(t)\right\rangle=0, \quad\left\langle\Omega^{\mathrm{Br}}(t) \Omega^{\mathrm{Br}}\left(t^{\prime}\right)\right\rangle=2 k_{B} T R \delta\left(t-t^{\prime}\right),
$$

where $R$ is the local hydrodynamic rotational mobility.

Unconfined or deep subphase: The translational velocity at a point $\mathbf{r}=r \hat{\mathbf{r}}$ away from an inclusion atop a deep subphase is

$$
\mathbf{u}^{\text {deep }}=-\frac{\sigma}{4 \pi \eta_{s} r}\left(1-2 \cos ^{2} \theta\right) \hat{\mathbf{r}} .
$$

For such an inclusion in an unconfined viscous membrane with $r \ll \ell$, the mobility is approximately [2,3] $M=1 / 4 \pi \eta_{s}$. Substituting in Eq. (1), using Eq (4), and assuming a constant dipole strength $\sigma=F L$, the translational velocity of each inclusion becomes:

$$
\left.\frac{d \mathbf{x}_{i}}{d t}\right|_{\text {deep }}=\sum_{j \neq i}^{N} \frac{\sigma}{4 \pi \eta_{s} r}\left(2 \cos ^{2} \theta_{i j}-1\right) \hat{\mathbf{r}}_{i j}+\sqrt{\frac{k_{B} T}{2 \pi \eta_{s} \Delta t}} \mathbf{w} .
$$

Here $\hat{\mathbf{r}}_{i j}$ is the unit vector pointing from the inclusion that is the source of the disturbance (with index $j$ ) to the current inclusion (with index $i$ ), and $\theta_{i j}$ is the polar angle in this frame of reference. Written this way, $\Delta t$ is the simulation time step and $\mathbf{w}$ is a white noise vector containing random numbers from a distribution of zero mean and unit variance. Nondimensionalizing Eq. (7) over $L$ for length and $\eta_{s} L^{2} / \sigma$ for time gives

$$
\left.\frac{d \tilde{\mathbf{x}}_{i}}{d \tilde{t}}\right|_{\text {deep }}=\sum_{j \neq i}^{N} \frac{1}{4 \pi \tilde{r}}\left(2 \cos ^{2} \theta_{i j}-1\right) \hat{\mathbf{r}}_{i j}+\sqrt{\frac{\tau}{2 \pi \Delta \tilde{t}}} \mathbf{w},
$$


where

$$
\tau=\frac{k_{B} T}{\sigma}
$$

is a dimensionless temperature. Alternatively, $\tau$ can be interpreted as (the inverse of) a Peclet number that compares convective flow due to the force dipole $(\propto \sigma)$ against Brownian diffusion. Similarly, the net rotational velocity of inclusion $i$, using a local rotational drag of $R=1 / 4 \pi \eta_{s} L^{2}[2,3]$ and nondimensionalizing like before is

$$
\left.\frac{d \tilde{\theta}_{i}}{d t}\right|_{\text {deep }}=\sum_{j \neq i}^{N}-\frac{1}{2 \pi \tilde{r}^{2}} \sin 2 \theta_{i j}+\sqrt{\frac{\tau}{2 \pi \Delta \tilde{t}}} \mathbf{w}
$$

Numerically integrating the coupled system of Eqs. (8) and (10) in a 2D periodic box gives the results in Figure 4.

Confined or shallow subphase: In this case, the translational disturbance velocity around an active inclusion is

$$
\mathbf{u}^{\text {confined }}=-\frac{\sigma H}{\pi \eta r^{3}}\left[\left(1-4 \cos ^{2} \theta\right) \hat{\mathbf{r}}+2 \cos \theta \hat{\mathbf{p}}\right],
$$

The $2 \mathrm{D}$ isotropic membrane mobility is now $M=H / 2 \pi \eta R^{2}[2,4]$, so the net velocity of each inlcusion becomes

$$
\left.\frac{d \mathbf{x}_{i}}{d t}\right|_{\text {confined }}=\sum_{j \neq i}^{N}-\frac{\sigma H}{\pi \eta r^{3}}\left[\left(1-4 \cos ^{2} \theta_{i j}\right) \hat{\mathbf{r}}_{i j}+2 \cos \theta_{i j} \hat{\mathbf{p}}_{j}\right]+\sqrt{\frac{k_{B} T H}{\pi \eta R^{2} \Delta t}} \mathbf{w},
$$

where now $\mathbf{p}_{j}$ is the orientation of dipole that generates the disturbance. Nondimensionalizing in this case with a characteristic time $\eta L^{4} / \sigma H$, one finds

$$
\left.\frac{d \tilde{\mathbf{x}}_{i}}{d \tilde{t}}\right|_{\text {confined }}=\sum_{j \neq i}^{N}-\frac{1}{\pi \tilde{r}^{3}}\left[\left(1-4 \cos ^{2} \theta_{i j}\right) \hat{\mathbf{r}}_{i j}+2 \cos \theta_{i j} \hat{\mathbf{p}}_{j}\right]+\frac{1}{\sqrt{\pi s \Delta \tilde{t}}} \mathbf{w} .
$$

The corresponding angular velocity has no contribution due to dipolar interactions (as the confined flow is irrotational); rotation occurs due to Brownian fluctuations alone. Using a confined mobility of $R=2 H / \pi \eta L^{4}[4]$, the rotational velocity in dimensionless terms is

$$
\left.\frac{d \tilde{\theta}_{i}}{d t}\right|_{\text {confined }}=\sqrt{\frac{4 \tau}{\pi \Delta \tilde{t}}} \mathbf{w} .
$$

Numerically integrating the coupled system of Eqs. (13) and (14) gives the results in Figure 4 corresponding to a confined membrane.

Steric repulsion: Particle overlap is prevented in simulations by adding a soft repulsion when particles are close to each other. This effectively amounts to an additional pair-wise relative velocity of the form

$$
\left.\mathbf{u}_{i}\right|_{\text {steric }}=U_{s} \frac{e^{-\beta(r-L)}}{1+e^{-\beta(r-L)}} \hat{\mathbf{r}}_{i j},
$$

where $U_{s}$ is the contact velocity that decays exponentially over a length scale $\beta^{-1} \ll L$, ensuring that hydrodynamics dominates at all distances except near contact.

[1] N. Oppenheimer and H. Diamant, Biophysical Journal 96, 3041 (2009).

[2] H. Manikantan and T. M. Squires, Journal of Fluid Mechanics 892, P1 (2020).

[3] P. G. Saffman, Journal of Fluid Mechanics 73, 593 (1976).

[4] E. Evans and E. Sackmann, Journal of Fluid Mechanics 194, 553 (1988). 